\title{
Lyme disease: How reliable are serologic results?
}

\author{
Dan Gregson MD, G. Evans MD, David Patrick MD, William Bowie MD
}

See also page 1222 and www.cmaj.ca/lookup/doi/10.1503/cmaj.141413

$\mathrm{L}$ yme disease is a tick-transmitted bacterial infection that is well established in North America. It is uncommon in most areas of Canada, but its incidence and geographic range are increasing. The accurate diagnosis of Lyme disease is critical to ensure that those patients who truly have the condition are given appropriate antibiotics. Furthermore, an accurate diagnosis ensures patients with nonspecific symptoms are not mistakenly told that they have Lyme disease. In their recent practice article, ${ }^{1}$ Andany and colleagues discuss a clinical scenario in which a Canadian man pursued testing for Lyme disease through a commercial laboratory in the United States. The test showed a positive result that was at odds with serologic testing conducted through a public health laboratory. ${ }^{1}$

This patient scenario illustrates for readers that American specialty laboratories should not be considered to provide a more sensitive assay for the diagnosis of Lyme disease than their public health counterparts. Recent research has documented a high rate of false-positive results with extremely poor positive predictive value in some specialty laboratories. ${ }^{2}$ Mistakes in diagnosis can deprive patients of treatment specific to the true cause of their symptoms, and can result in prolonged therapy for a condition they do not have.

The methods for diagnosing Borrelia burgdorferi infection, the organism that causes Lyme disease, have been continuously improving since the microbe was first discovered in $1982 .^{3}$ The method involves the use of European Borrelia species and flagellar antigens in the screening serology to improve the sensitivity or negative predictive value, and confirmatory Western blotting assays to increase the specificity and positive predictive value of the test. ${ }^{4,5}$ Some Lyme disease advocacy groups espouse that Centers for Disease Control and Prevention (CDC) criteria used for the serologic diagnosis of Lyme disease are inadequate, and they recommend alternative interpretive standards. ${ }^{6}$ However, a recent study by Fallon and colleagues ${ }^{2}$ formally evaluated how current testing algorithms work in two patient groups and several types of laboratories in the US. The findings support previous conclusions of the $\mathrm{CDC}^{7}$ and highlight two important lessons for physicians and consumers.

In a well-defined cohort of patients with posttreatment symptoms of Lyme disease, tests done in a university or commercial laboratory using well-defined CDC criteria for the serologic diagnosis of Lyme disease were as sensitive as testing done in laboratories specializing in Lyme testing. This remained true even when the specialty laboratories used in-house criteria to "increase" the sensitivity of their Western blot testing. Accordingly, such laboratories cannot be considered to be better at picking up infections missed by standard CDC criteria.

Furthermore, 40 patients without Lyme disease were included in the study as a negative control group. The inclusion of immunoglobulin $\mathrm{M}$ in the interpretation of control group Western blot samples led to false-positive results from three of the four laboratories studied (a rate of $2.5 \%-25 \%$ ). One specialty laboratory using inhouse criteria (immunoglobulins $\mathrm{G}$ or $\mathrm{M}$ ) had false-positive results in $57 \%$ of the samples from the negative control group.

Fallon and colleagues' study further dispels the myth that US specialty laboratories provide a more sensitive assay for the diagnosis of Lyme disease, and documents a high rate of falsepositive results with poor positive predictive values in some specialty laboratories. As a conse-

\section{KEY POINTS}

- The serologic diagnosis of Lyme disease in Canada is best done using standard laboratory protocols as implemented by the National Microbiology Laboratory of Canada using criteria recommended by the Centers for Disease Control and Prevention.

- Recent evidence suggests that standard assays and testing algorithms used in Canada are as sensitive as those used in American specialty laboratories for detecting infection with Borrelia burgdoferi.

- Specialty laboratory tests have a high rate of false-positive results owing to their use of non-evidence based interpretation criteria, particularly when results rely solely on Western blot analysis.

- Most Canadians who are told that they have Lyme disease based solely on results from specialty laboratory typically have other causes for their symptoms. 
quence, patients and physicians should be cautious in choosing a referral laboratory in the US when seeking "second opinion" serology after receiving a negative test result in Canada. Laboratories that use the standard CDC two-tier testing algorithms should be preferred over those that report results based on unproven, unvalidated, in-house criteria. Any positive result from a test that relies solely on Western blotting is most likely a false-positive.

Patients with chronic subjective symptoms without a diagnosis can be vulnerable and desperate for an answer as to the cause of their illness. Giving them a false diagnosis based on flawed testing is misleading. Inappropriate therapy based on such results leads to economic, psychological and physical adverse outcomes. ${ }^{8-10}$ Rather, these patients deserve a complete and accurate evaluation to detect illnesses for which appropriate interventions can be applied and, whatever their diagnosis, supports to improve the quality of life for themselves and their families.

\section{References}

1. Andany N, Cardew S, Bunce PE. A 35-year-old man with a positive Lyme test result from a private laboratory. CMAJ 2015;187:1222-4

2. Fallon BA, Pavlicova M, Coffino SW, et al. A comparison of Lyme disease serologic test results from 4 laboratories in patients with persistent symptoms after antibiotic treatment. Clin Infect Dis 2014;59:1705-10.
3. Burgdorfer W, Barbour AG, Hayes SF, et al. Lyme disease-a tick-borne spirochetosis? Science 1982;216:1317-9.

4. Branda JA, Strle F, Strle K, et al. Performance of United States serologic assays in the diagnosis of Lyme borreliosis acquired in Europe Clin Infect Dis 2013;57:333-40.

5. Wormser GP, Tang AT, Schimmoeller NR, et al. Utility of serodiagnostics designed for use in the United States for detection of Lyme borreliosis acquired in Europe and vice versa. Med Microbiol Immunol (Berl) 2014;203:65-71.

6. Basic infromation about Lyme disease. Bethesda (MD): International Lyme and Associated Diseases Society. Available: www. ilads.org/lyme/about-lyme.php (accessed 2015 Aug. 6).

7. Notice to readers: caution regarding testing for Lyme disease. Atlanta: Centers for Disease Control and Prevention; 2005: Available: www.cdc.gov/mmwr/preview/mmwrhtml/mm5405a6 .htm (accessed 2015 Aug. 5).

8. Sigal LH. The Lyme disease controversy. Social and financial costs of misdiagnosis and mismanagement. Arch Intern Med 1996; 156:1493-500.

9. Cottle LE, Mekonnen E, Beadsworth MBJ, et al. Lyme disease in a British referral clinic. QJM 2012;105:537-43.

10. White B, Seaton RA, Evans TJ. Management of suspected Lyme borreliosis: experience from an outpatient parenteral antibiotic therapy service. QJM 2013;106:133-8.

Affiliations: Division of Infectious Diseases (Bowie), University of British Columbia, Vancouver, BC; Association of Medical Microbiology and Infectious Disease Canada (Evans), Department of Medicine, Queen's University, Kingston, Ont.; School of Population and Public Health (Patrick), University of British Columbia, Vancouver, BC; Association of Medical Microbiology and Infectious Disease Canada (Gregson), Department of Pathology and Laboratory Medicine, University of Calgary, Calgary, Alta.

Contributors: All of the authors conceived the original idea for the article. Dan Gregson drafted the article. All of the authors revised the article for important intellectual content. All of the authors approved the final version to be published and agreed to act as guarantors of the work. 